\title{
A complex approach to the solution of problems in mechanics of deformable rigid bodies
}

\author{
Murad Agakhanov ${ }^{1, *}$ and Elifkhan Agakhanov ${ }^{2}$ \\ ${ }^{1}$ Moscow State University of Civil Engineering, 26, Yaroslavskoye sh., Moscow, 129337, Russia \\ ${ }^{2}$ Dagestan State Engineering University, 367015, 70, Imam Shamil Avenue, Makhachkala, Russia
}

\begin{abstract}
There exists an opinion that the modern numerical methods allow to solve practically any problem in mechanics. But it should be noted that both analytical and experimental methods, as before, are urgent, and exactly a complex of methods develops the mechanics of deformable rigid bodies. The statement of a problem in displacements for some possible cases of equivalent substitution of loads allows to formulate necessary and sufficient conditions of existence of an analogy presenting the effect of a forced deformation in the form of the sum of surface and volume forces, the effect of volume forces in the form of the sum of surface forces and forced deformations, the effect of surface forces in the form of the sum of forced deformations and volume forces. The substitution of volume forces for surface loads and forced deformations allows to extend the use of experimental methods and often to solve through an experimental-andtheoretical approach the problems, which cannot be solved through other methods. The obtained results are a considerable step in the development of one of the approaches combining experimental, analytical and numerical methods of solution of linear problems in mechanics of deformable rigid bodies.
\end{abstract}

\section{Introduction}

The practical construction always enriches itself by new exact experimental-andtheoretical knowledge, and the volume of active information resources with respect to the general volume of accumulated professional knowledge extends much. In this connection, the solution of problems in mechanics of deformable rigid bodies concerning the total use of the material strength for the purpose of achievement of minimum cost and minimum specific consumption of materials for structural elements of buildings and structures widely use the computer facilities. Arguing against the opinion that the modern numerical methods allow to solve practically any problem in mechanics, we should note that the analytical and experimental methods, as before, are urgent, and exactly a complex of methods develops the mechanics of deformable rigid bodies $[1,2]$. Nowadays, one of the most important development trends in mechanics of deformable rigid bodies is the creation of approaches which allow to combine huge computing potentialities of modern super-computers with experimental methods of study of materials and structures.

\footnotetext{
* Corresponding author: muradak@mail.ru
} 
In the static problem in mechanics of deformable rigid bodies, all the forces, depending on their presence in the resolving set of equations, are subdivided into surface forces $\mathrm{P}_{\mathrm{i}}$, volume forces $\mathrm{F}_{\mathrm{i}}$ and distortions or forced deformation $\xi$.

There exists a partial equivalence of effects consisting of the identity (analogy) of stresses or displacements under the effect of different loads [3, 4]. Some works consider the analogy of the effect of volume and surface forces which exists only under strict constraints $[5,6]$.

\section{State of the problem}

This article formulates necessary and sufficient conditions of the existence of the analogy which allows to present the effect of a forced deformation as the sum of effects of surface and volume forces, the effect of volume forces as the sum of effects of surface forces and forced deformations, the effect of surface forces as the sum of forced deformations and volume forces. This analogy is important for the experimental solution of problems, as the feasibility of every effect is restricted to the potentialities of the modelling methods. Let us consider a body of arbitrary shape occupying the volume $V$ with the surface $B=B_{1}+B_{2}$. On the surface area $B_{1}$, the displacements $f_{i}$ are given; on the surface area $B_{2}$ there act some surface loads and there is a system of restraints. The body is subject to the effect of surface loads, volume forces and forced deformations.

The displacements $U_{i}$ in the domain $V+B$, independent of the domain connectivity, satisfy to the equations:

$$
U_{i, j j}+U_{j, i j}+2 S_{, i}+\frac{F_{i}}{G}=0 \text { in } V
$$

$U=f_{i}$ on $B_{l}$ (this condition remains in all following considerations);

$$
2 G\left[0,5\left(U_{i, j}+U_{j, i}\right)+\mathrm{d}_{i j} S\right] n_{j}=P_{i} \text { on } B_{2},
$$

where

$$
S=\frac{\mathrm{n}}{E} \mathrm{~s}_{i i}-\mathrm{x}_{\text {in }} V
$$

With $v \neq 0.5$ this dependence takes the form:

$$
S=\frac{\mathrm{n}}{1-2 \mathrm{n}} U_{j, j}-\frac{1+\mathrm{n}}{1-2 \mathrm{n}} \mathrm{x}
$$

\section{Research result}

Let us consider the possible cases of equivalent substitution of loads.

1. It is required to determine the stress-and-strain state caused only by the forced deformation $\xi$.

The equations (1) and conditions (2) in this case have the form

$$
\begin{gathered}
U_{i, j j}^{(\mathrm{x})}+U_{j, i j}^{(\mathrm{x})}+2 S_{, i}^{(\mathrm{x})}=0 \text { in } V \\
2 G\left[0,5\left(U_{i, j}^{(\mathrm{x})}+U_{j, i}^{(\mathrm{x})}\right)+\mathrm{d}_{i j} S^{(\mathrm{x})}\right] n_{j}=0 \text { on } B_{2}
\end{gathered}
$$


Let the researcher have some methods which allow to determine the stress-and-strain state caused by the effect of surface forces $P_{i}$ and volume forces $F_{i}$.

The equations (1) and conditions (2) will take the form:

$$
\begin{gathered}
U_{i, j j}^{(P, F)}+U_{j, i j}^{(P, F)}+2 S_{, i}^{(P, F)}+\frac{F_{i}}{G}=0 \text { in } V \\
2 G\left[0,5\left(U_{i, j}^{(P, F)}+U_{j, i}^{(P, F)}\right)+\mathrm{d}_{i j} S^{(P, F)}\right] n_{j}=P_{i} \text { on } B_{2} .
\end{gathered}
$$

The conditions of the identity of displacements $U_{i}^{(\mathrm{x})} \equiv U_{i}^{(P, F)}$ are the following:

$$
\begin{gathered}
2 S_{, i}^{(\mathrm{x})}=2 S_{, i}^{(P, F)}+\frac{F_{i}}{G} \text { in } V \\
2 G S^{(\mathrm{x})} n_{i}=2 G S^{(P, F)} n_{i}-P_{i} \text { on } B_{2} .
\end{gathered}
$$

Further, with consideration of (4), we obtain

$$
\begin{gathered}
P_{i}=\frac{E}{1-2 \mathrm{n}} \times n_{i} \\
F_{i}=-\frac{E}{1-2 \mathrm{n}} \mathrm{x}_{, i}
\end{gathered}
$$

From the expression (11) follows that the differentiability of the forced deformation function $\xi$ in $V$ and its continuity on $B_{2}$ is a necessary and sufficient condition of applicability of the analogy concerned.

The stresses caused by the forced deformations $\xi$ are determined by the formulas

$$
\mathrm{s}_{i j}^{(\mathrm{x})}=2 G\left[0,5\left(U_{i, j}^{(\mathrm{x})}+U_{j, i}^{(\mathrm{x})}\right)+\mathrm{d}_{i j} S^{(\mathrm{x})}\right]
$$

The stresses caused by the substituting loads $F_{i}, P_{i}$ are

$$
\mathbf{s}_{i j}^{(P, F)}=2 G\left[0,5\left(U_{i, j}^{(P, F)}+U_{j, i}^{(P, F)}\right)+\mathrm{d}_{i j} S^{(P, F)}\right]
$$

With $U_{i}^{(\mathrm{x})} \equiv U_{i}^{(P, F)}$ we have

$$
\mathrm{S}_{i j}^{(\mathrm{x})}=\mathrm{s}_{i j}^{(P, F)}+\mathrm{d}_{i j} 2 G\left[S^{(\mathrm{x})}-S^{(P, F)}\right]
$$

With consideration of (4) we have

$$
\mathrm{s}_{i j}^{(\mathrm{x})}=\mathrm{s}_{i j}^{(P, F)}-\mathrm{d}_{i j} \frac{E}{1-2 \mathrm{n}} \mathrm{x}
$$

This result is well known as the analogy by S. P. Timoshenko; it is used for the substantiation of the experimental method of "defrosting" of free temperature strains $[6,7]$ which is applied for the solution of the thermo-elasticity problem. 
2. Without loss of generality, we consider the possibility of presentation of a stress-andstrain state of a body under the effect of a given surface load $P_{i}$ in the form of the sum of stress-and-strain states caused by the effect of volume forces $F_{i}$ and forced deformations $\xi$.

The initial set of equations and the boundary conditions are

$$
\begin{gathered}
U_{i, j j}^{(P)}+U_{j, i j}^{(P)}+2 S_{, i}^{(P)}=0 \text { in } V \\
2 G\left[0,5\left(U_{i, j}^{(P)}+U_{j, i}^{(P)}\right)+\mathrm{d}_{i j} S^{(P)}\right] n_{j}=P_{i} \text { on } B_{2} .
\end{gathered}
$$

The set of equations and the boundary conditions for the substituting loads

$$
\begin{gathered}
U_{i, j j}^{(F, \mathrm{x})}+U_{j, i j}^{(F, \mathrm{x})}+2 S_{, i}^{(F, \mathrm{x})}+\frac{F_{i}}{G}=0 \text { in } V ; \\
2 G\left[0,5\left(U_{i, j}^{(F, \mathrm{x})}+U_{j, i}^{(F, \mathrm{x})}\right)+\mathrm{d}_{i j} S^{(F, \mathrm{x})}\right] n_{j}=0 \text { on } B_{2} .
\end{gathered}
$$

The conditions of identity of displacements $U_{i}^{(P)} \equiv U_{i}^{(F, \xi)}$ after the transformations similar to (9) and (10) take the form

$$
\begin{gathered}
P_{i}=\frac{E}{1-2 \mathrm{n}} \mathrm{x} n_{i} \\
F_{i}=\frac{E}{1-2 \mathrm{n}} \mathrm{x}_{, i}
\end{gathered}
$$

The conditions of applicability of the analogy here are the same (see point 1).

The stresses are determined through the formulas

$$
\mathrm{s}_{i j}^{(P)}=\mathrm{s}_{i j}^{(F, \mathrm{x})}+\mathrm{d}_{i j} 2 G\left[S^{(P)}-S^{(F, \mathrm{x})}\right]=\mathrm{s}_{i j}^{(F, \mathrm{x})}+\mathrm{d}_{i j} \frac{E}{1-2 \mathrm{n}} \mathrm{x}
$$

3. The most interesting opportunity is the expression for a stress-and-strain state caused by the given volume forces $F_{i}$ in the form of a stress-and-strain state caused by the total effect of forced deformations $\xi$ and surface loads $P_{i}$.

The set of equations and the boundary conditions have the form

$$
\begin{gathered}
U_{i, j j}^{(F)}+U_{j, i j}^{(F)}+2 S_{, i}^{(F)}+\frac{F_{i}}{G}=0 \text { in } V ; \\
2 G\left[0,5\left(U_{i, j}^{(F)}+U_{j, i}^{(F)}\right)+\mathrm{d}_{i j} S^{(F)}\right] n_{j}=0 \text { on } B_{2} .
\end{gathered}
$$

The set of equations and the boundary conditions for the substituting loads

$$
\begin{gathered}
U_{i, j j}^{(P, \mathrm{x})}+U_{j, i j}^{(P, \mathrm{x})}+2 S_{, i}^{(P, \mathrm{x})}=0 \text { in } V ; \\
2 G\left[0,5\left(U_{i, j}^{(P, \mathrm{x})}+U_{j, i}^{(P, \mathrm{x})}\right)+\mathrm{d}_{i j} S^{(P, \mathrm{x})}\right] n_{j}=P_{i} \text { on } B_{2} .
\end{gathered}
$$


The conditions of the identity of displacements $U_{i}^{(F)} \equiv U_{i}^{(P, \xi)}$ after the transformations similar to (9) and (10) take the form

$$
\begin{aligned}
& \frac{E}{1-2 \mathrm{n}} \mathrm{x}_{, i}=-F_{i} \\
& \frac{E}{1-2 \mathrm{n}} \mathrm{x} n_{i}+P_{i}=0
\end{aligned}
$$

it follows from this that the condition of the applicability in this case is the potentiality of volume forces $F_{i}$ in $V$ and the continuity of their potential on $B_{2}$.

The stresses in this case are determined by the formula

$$
\mathrm{s}_{i j}^{(F)}=\mathrm{s}_{i j}^{(\mathrm{x}, P)}+\mathrm{d}_{i j} 2 G\left[S^{(F)}-S^{(\mathrm{x}, P)}\right]=\mathrm{s}_{i j}^{(\mathrm{x}, P)}+\mathrm{d}_{i j} \frac{E}{1-2 \mathrm{n}} \mathrm{x}
$$

In this form, the analogy may be used for the process of modelling the volume forces caused by the potential fields of the type of equilibrium pressure and percolation pressure, electrostatic and magnetic fields, centrifugal accelerations etc.

With respect to the methodology, the methods of experimental determination of stresses and displacements under the effect of forced deformations and surface loads to which the problem is reduced have been developed with a completeness sufficient for the deduction of efficient solutions.

Let us consider some particular cases of application of the analogy.

1. Let us consider a body statically determinate with respect to external forces under the effect of a volume force $F_{i}=$ const. In this case, the substituting loads $\xi$ and $P_{i}$ are linear functions of coordinates; the displacements are presented by the formula

$$
U_{i}^{(F)}=U_{i}^{(\mathrm{x})}+U_{i}^{(P)}
$$

A linear distribution of forced deformations does not cause any stresses, therefore the formula (30) takes the form

$$
\mathrm{s}_{i j}^{(F)}=\mathrm{s}_{i j}^{(P)}+\mathrm{d}_{i j} \frac{E}{1-2 \mathrm{n}} \mathrm{x}=\mathrm{s}_{i j}^{(P)}-\mathrm{d}_{i j} P
$$

In particular, the formula (32) corresponds to the well-known analogy by

S. G. Gutman [3], when $F_{i}$ is the gravitational force. There is a widespread term "BiotGutman analogy", but M. Biot considered the application of the analogy only for a plane problem [8].

2. Consider a body from an incompressible material $(v=0.5)$. In this case, the resolving set of equations includes not only (1) and (2), but also an additional condition [9]

$$
U_{j, j}=3 \mathrm{x}
$$

or, in the absence of forced deformations,

$$
U_{j, j}=0
$$

To establish an analogy in this case, it is necessary to have

$$
\mathrm{x} \equiv 0
$$


from which it follows that for an incompressible body the analogy by S. P. Timoshenko is inapplicable. In this case, it is expedient to use the method suggested by S. E. Bugaenko [6].

Consider the use of an analogy for an incompressible body under the effect of volume forces $F_{i}$. As a substituting load, we consider the surface load $P_{i}$.

The resolving set of equations and the boundary conditions, respectively, take the form (24) - (27) with the simplifications stipulated by the condition (34).

The conditions of the identity $U_{i}^{(F)} \equiv U_{i}^{(P)}$ take the form

$$
\begin{gathered}
2 S_{, i}^{(F)}+\frac{F_{i}}{G}=2 S_{, i}^{(P)} \text { in } V \\
2 G S^{(F)} n_{i}=2 G S^{(P)} n_{i}-P_{i} \text { on } B_{2},
\end{gathered}
$$

from what it follows that

$$
P_{, i}=F_{i}
$$

Thus, the well-known analogies existing under strict restraints are only particular cases of the application of the equivalence of effects established in this article.

\section{Conclusions}

The substitution of volume forces for surface loads and forced deformations allows to expand the application of experimental methods and, often, to solve through an experimental-and-theoretical approach the problems, the solution of which by other methods is either difficult or impossible $[10,11]$. The results obtained in this article are a considerable step in the process of development of an approach combining experimental, analytical and numerical methods of solution of linear problems in mechanics of deformable rigid bodies, it being known that the main effect is not the reduction of volume of calculations (which is not a question of principle nowadays), but the construction of a proper interpretation of the results containing large data bases; the interpretation which opens the possibility of a proper analysis of obtained solutions and of comprehension of the problem which allows to solve the problem of strength, reliability and efficiency of products.

\section{References}

1. E.K. Agakhanov, Bulletin of Dagestan State Engineering University. Engineering Sciences 2 (2013)

2. G.E. Agakhanov, About mathematical modeling of physical effects in soils. Science revue 12 (2014)

3. S.G. Gutman, Proceedings of the Hydro-engineering Research Institute 11 (1934)

4. N.F. Lebedev, Applied mechanics 2 (1977)

5. S.P. Timoshenko, J. Goudier, Theory of elasticity (Nauka, Moscow, 1975)

6. S.E. Bugaenko, Proceedings of the Academy of Sciences of the USSR, Mechanics of rigid bodies 4 (1980)

7. G.L. Khessin, Photo-elasticity method (Stroyizdat, Moscow, 1975) 
8. M.A. Biot, Trans. ASME. Appl. Mech. 2, 57 (1935)

9. E.K. Agakhanov, M.K. Agakhanov, Industrial and Civil Construction 11 (2015)

10. G.E. Agakhanov, Bulletin of Dagestan State Engineering University. Engineering sciences 3 (2015)

11. E.K. Agakhanov, M.K. Agakhanov, MGSU Bulletin 3, 4 (2010) 